\title{
Terapias conductuales de tercera generación en personas mayores: una revisión de la literatura
}

\author{
Luis Jorge Ruiz Sánchez* y Zaida Callejón Ruiz \\ Universidad de Almería, España
}

(Recibido 2 Julio, 2014; Aceptado 5 Septiembre, 2014)

RESUMEN:El objetivo de este estudio fue analizar el grado de evidencia clínica de las Terapias Conductuales de Tercera Generación en la población mayor a través de una revisión de los estudios más relevantes en la literatura científica. De un total de 332 referencias identificadas, 16 estudios cumplieron el criterio de selección. El análisis de estos estudios mostró que las intervenciones basadas en mindfulness acumulan mayor evidencia, seguidas por la Terapia de Aceptación y Compromiso (ACT) y la Activación Conductual (BA). Sin embargo, los resultados son limitados. Son necesarios más estudios controlados aleatorizados para clarificar la eficacia de este tipo de tratamientos.

Palabras clave: personas mayores, revisión, conciencia plena, terapia de aceptación y compromiso, activación conductual, terapia dialéctico-conductual.

Third generation of behavior therapies with older adults: a review of literature.

ABSTRACT:The aim of this study was to determine the degree of clinical evidence of Third Generation of Behavior Therapies with older adults from a review of the most relevant studies in the scientific literature. From a total of 332 references identified, 16 studies met the selection criteria. The analysis of these studies showed that intervention based on Mindfulness accumulates more evidence, followed by Acceptance and Commitment Therapy (ACT) and Behavior Activation (BA). Nevertheless, the results are limited. More randomized controlled trials are needed to clarify the efficacy of this kind of treatments.

Keywords: older adults, review, mindfulness, acceptance and commitment therapy, behavior activation, dialectical behavior therapy .

\section{INTRODUCCIÓN}

La mayoría de aproximaciones terapéuticas a la población mayor se basan en intervenciones psicosociales, cognitivo-conductuales, de solución de problemas y/o programación de actividades (Areán y Cook, 2002; Mackin y Areán, 2005). Este tipo de intervenciones presentan un marcado énfasis en el cambio o control de los pensamientos como eje causal del problema, una excesiva importancia del bienestar de tipo hedónico, una tendencia a estandarizar los tratamientos y un estilo eminentemente psicoeducativo (véase Petkus y Wetherell, 2013;Márquez, Fernández, Romero y Losada, 2013). 
Durante los últimos años, han emergido un amplio número de terapias psicológicas que mantienen una aproximación radicalmente distinta a los problemas del ser humano. Tales terapias presentan una orientación marcadamente empírica, con un acento en los principios del aprendizaje. El objetivo de estas terapias se centra en alterar la función de los pensamientos, recuerdos, emociones u otros eventos privados a través del contexto en el que estos síntomas resultan problemáticos a fin de construir repertorios flexibles y efectivos (Hayes, 2004; Luciano y Valdivia, 2006). Como forma de agrupar este nuevo conjunto de terapias han sido denominadas Terapias de Tercera Generación o Terapias Contextuales (p. ej., Hayes, 2004, Pérez-Álvarez, 2012). Entre estas terapias figuran las Terapias basadas en Mindfulness (MBT), la Terapia de Activación Conductual (BA), la Terapia Dialéctico-Conductual (DBT), la Psicoterapia Analítico-Funcional (FAP) y la Terapia de Aceptación y Compromiso (ACT).

Terapias basadas en Mindfulness (MBT). Entre las MBT podemos encontrar la Terapia Cognitiva basada en Mindfulness (MBCT; Segal, Williams y Teasdale, 2002) o la Terapia de Reducción de Estrés basada en Mindfulness (MBSR; Kabat-Zinn, 1990). El origen de este tipo de terapias recae en el Budismo y existe una gran variedad de técnicas como la Meditación Trascendental (MT) o la Meditación Mindfulness (MM) que han sido incorporadas a las mismas. Las MBT promueven la habilidad para centrarse en la experiencia del momento presente, haciendo énfasis en notar los eventos privados con una actitud de apertura, curiosidad y aceptación (Bishop et al., 2004). Para ello hacen uso de metáforas, poemas y ejercicios de meditación. Igualmente, se realizan ejercicios que permiten al individuo notar cómo surgen los pensamientos o emociones y cómo éstos llevan a diferentes estados emocionales o comportamientos.

Terapia de Activación Conductual (BA). Esta terapia está centrada en los componentes conductuales de las terapias cognitivas aplicadas a la depresión (Jacobson et al., 1996). El objetivo fundamental de la Activación Conductual es que el individuo sea capaz de observar la conexión entre su estado de ánimo y otros comportamientos, de forma que pueda desarrollar un plan para incrementar el número de actividades y experiencias que puedan caer bajo el reforzamiento positivo (Jacobson, Martell yDimidjian, 2001). Para ello utiliza procedimientos clásicos como la extinción, el moldeamiento, la exposición, el entrenamiento en habilidades sociales y resolución de problemas. Este abordaje se ha mostrado especialmente eficaz en la depresión (Ekers, Webster, Straten, Cuijpers, Richards y Gilbody, 2014).

Terapia Dialéctico-Conductual (DBT). La DBT fue desarrollada para el tratamiento del trastorno límite de la personalidad. Incluye elementos de la terapia cognitivo-conductual y de la filosofía Zen, centrándose en estrategias de aceptación y cambio conductual para mejorar las habilidades de regulación emocional (Linehan, 1993). La DBT utiliza técnicas basadas en la exposición, el manejo 
de contingencias y el entrenamiento en habilidades. A esto se suman técnicas basadas en mindfulness que implican observar y describir de forma consciente lo que acontece a fin de adquirir habilidades como la tolerancia al estrés, la regulación emocional y la eficacia interpersonal. Esta terapia se ha mostrado efectiva principalmente en trastornos de personalidad, depresión, comportamientos autolesivos e intentos de suicidio (Panos, Jackson, Hasan y Panos, 2014; Valentine, Bankoff, Poulin, Reidler y Pantalone, 2014).

Terapia Analítico-Funcional (FAP).FAP es una terapia centrada en el análisis de las contingencias que ocurren en el contexto terapéutico, en el reforzamiento y moldeamiento directo de conductas (Kohlenberg y Tsai, 1991). Para ello, la intervención está centrada en detectar y generar conductas clínicamente relevantes, reforzar las mejoras del cliente, analizar las propiedades reforzantes de las conductas del terapeuta sobre las del cliente e incrementar las interpretaciones que describen las relaciones funcionales implicadas en el comportamiento del cliente (para una revisión Kohlenberg y Tsai, 1991; Kohlenberg y Tsai, 1993)

Finalmente, la Terapia de Aceptación y Compromiso (ACT; Hayes, Strosahl y Wilson, 1999; Wilson y Luciano, 2002) es el modelo de intervención contextualista funcional más representativo de este movimiento y que cuenta con más evidencia empírica (Hayes, 2004; Ruiz, 2010). ACT tiene como objetivo alterar el Trastorno de Evitación Experiencial (TEE), entendido como la dimensión funcional a la base de los diferentes trastornos psicológicos. Para ello se emplean metáforas y ejercicios experienciales que permitan incrementar flexibilidad psicológica, generando un repertorio extenso y flexible de comportamientos inscritos en direcciones personalmente valiosas donde el malestar estaría incluido ( $\mathrm{p}$. ej., Luciano y Valdivia, 2006; Wilson y Luciano, 2002).

Si bien todas las aplicaciones terapéuticas mencionadas arriba presentan un nexo común, existen diferencias entre ellas entorno a su eficacia, los procedimientos clínicos utilizados o la población en la que han sido aplicadas. El objetivo del presente estudio es proporcionar una revisión sobre la evidencia empírica de las principales intervenciones en el mayor desde las Terapias de Tercera Generación.

\section{MÉTODO}

Se llevó a cabo una búsqueda en las bases de datos electrónicas Web of Science (WOS) desde 1960 hasta 2014 (incluyendo la colección principal de WOS, MEDLINE y SCIELO), se consultó en la base de datos SCOPUS (desde 1964 hasta 2014) y se complementó la búsqueda con una consulta manual de las referencias extraídas.

Para realizar la búsqueda se usaron operadores booleanos y truncamientos. En WOS se introdujeron las siguientes palabras clave: (Mindful* AND old*) OR 
(Mindful* AND Elder*); se procedió de igual forma con Acceptance and Commitment Therapy, Behavior Activation, Dialectial Behavior Therapy o Functional Analytic Psychotherapy. La búsqueda fue filtrada para ensayos clínicos.

En SCOPUS se utilizaron los siguientes operadores: (TITLE-ABS-KEY ("Dialectical Behavior Therapy" OR mindful* OR "Acceptance and commitment" OR "behavioral activation" OR "Functional Analytic Psychotherapy") AND (TITLE-ABS-KEY (old* OR elder* OR older adults). La búsqueda se limitó a las siguientes áreas: medicina, psicología, enfermería, ciencias sociales, artes y humanidades y profesiones de la salud.

Una vez seleccionados todos los estudios, se realizó un proceso de selección por dos revisores de acuerdo a los siguientes criterios: a) ser una intervención incluida dentro de las denominadas terapias de tercera generación; b) ser una muestra con diagnóstico clínico; y c) los participantes deben tener una edad media igual o superior a 60 años. Por tanto, se excluyeron estudios de caso, teóricos, metodológicos, correlacionales, cualitativos y/o protocolos experimentales sin muestra clínica. Se excluyeron del presente análisis el estudio de Lych, Morse, Mendelson y Robins (2003) y el de Gallagher y Thompson (1983) por presentar datos parcialmente repetidos (véase Gallagher y Thompson, 1982; Lynch et al., 2007).

\section{RESULTADOS}

Se observa un incremento en el número de publicaciones desde el 2007 que llega a su punto más alto en el 2014 (Figura 1).

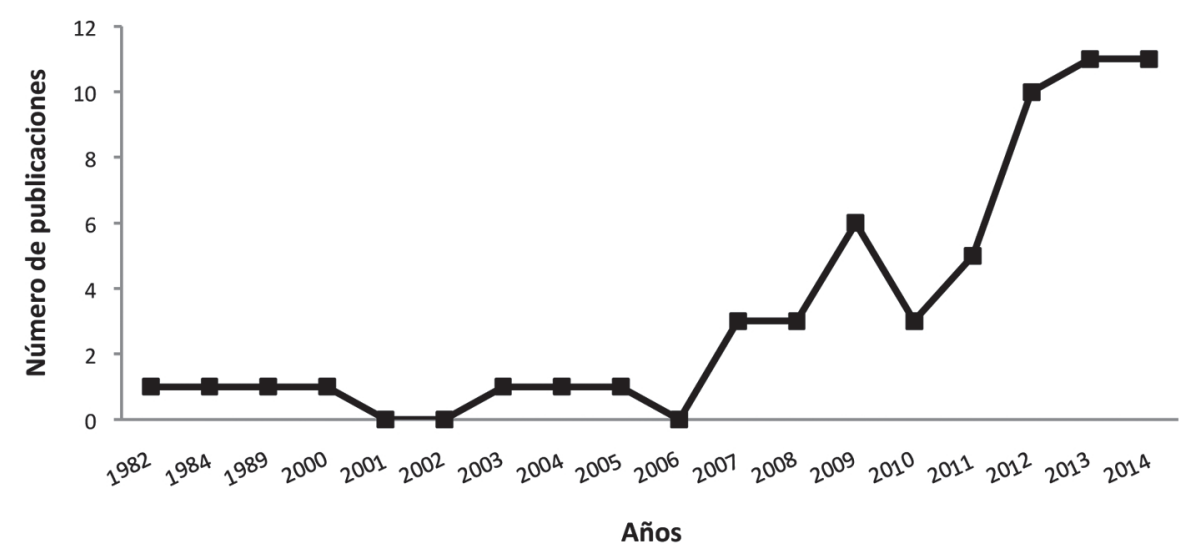

Figura 1. Distribución por años del número de publicaciones (estudios correlacionales, teóricos y empíricos) de las terapias conductuales de tercera generación en el mayor. 
La Figura 2 muestra el porcentaje de publicaciones en función del tipo de aproximación terapéutica en el mayor, incluyendo estudios correlacionales, teóricos y empíricos.

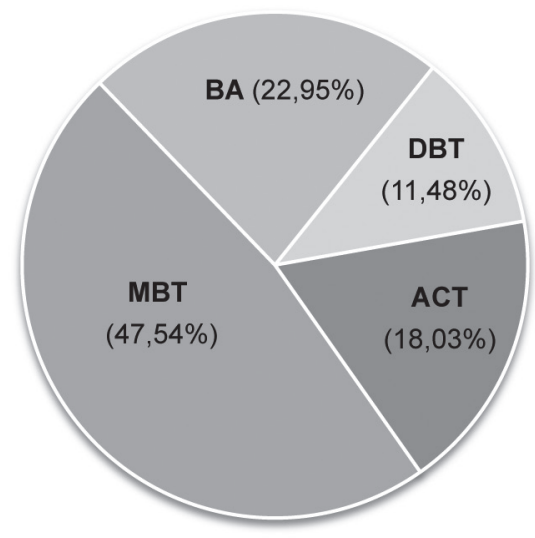

Figura 2. Desglose del porcentaje de publicaciones desde las diferentes aproximaciones terapéuticas entre los años 1982 y 2014. BA: Behavioral Activation; ACT: Acceptance and Commitment Therapy; DBT: Dialectical Behavior Therapy; MBT: Mindfulness-based Therapy.

De un total de 332 artículos encontrados, se seleccionaron 16 artículos para su análisis de acuerdo a los criterios de selección establecidos (Tabla 1). El 68,75\% de los estudios seleccionados son ensayos controlados aleatorizados y, del total, el 50\% presenta algún tipo de seguimiento. El 43,75\% de los ensayos clínicos incluidos corresponden a Mindfulness, el 25\% a la Terapia de Aceptación y Compromiso (ACT), el 18,75\% a la Terapia de Activación Conductual (BA) y el 12,5\% a la Terapia Dialéctico-Conductual (DBT). No se encontró ningún ensayo desde la Terapia Analítico-Funcional (FAP). 
Tabla 1. Descripción de los Ensayos Clínicos Aleatorizados y no Aleatorizados en Población Mayor con Diagnóstico Clínico.

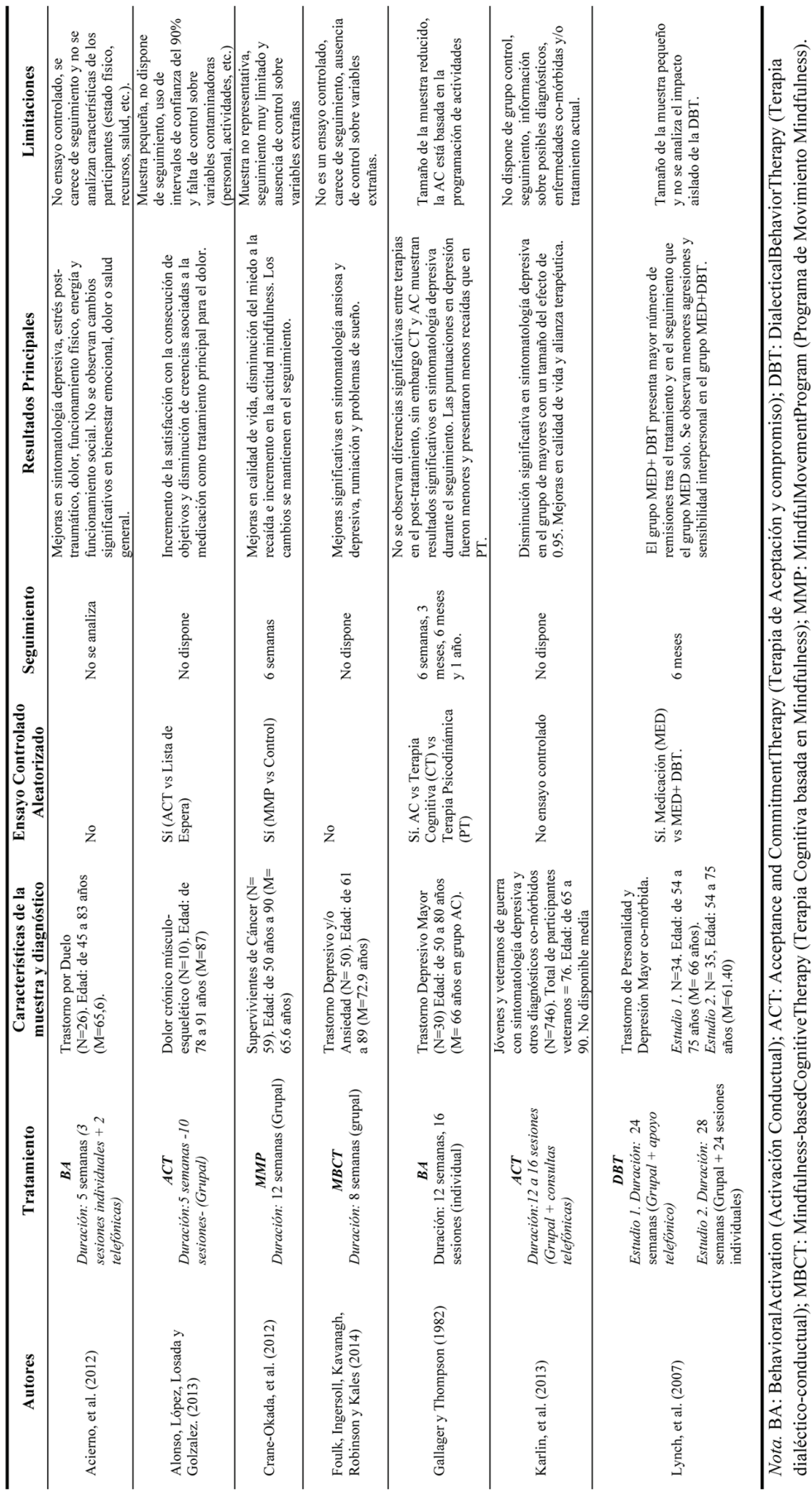




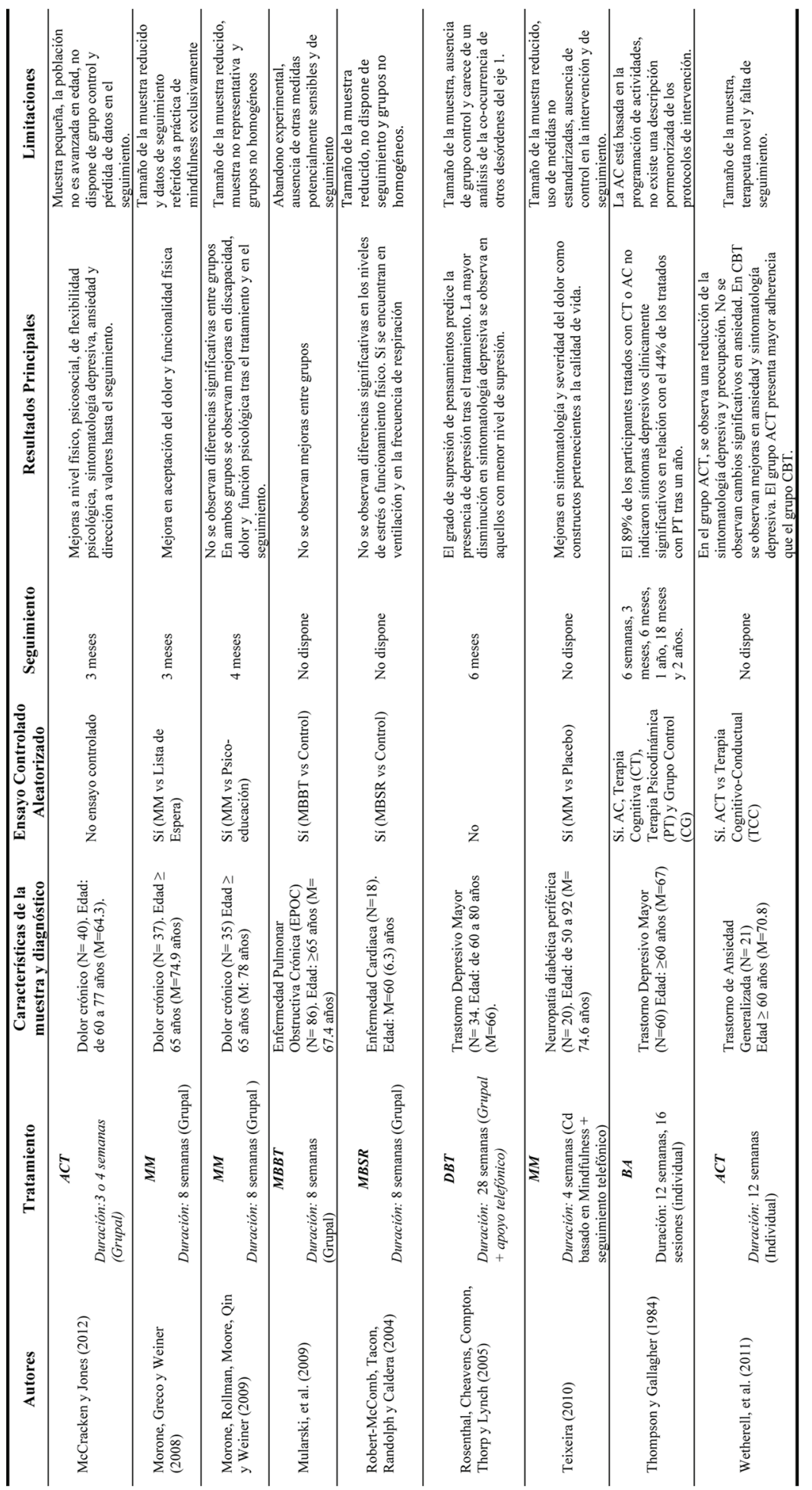




\section{DISCUSIÓN}

Las denominadas Terapias de Tercera Generación están emergiendo como una alternativa de intervención eficaz para los diferentes problemas que pueden acontecer en la población mayor. En concreto, este tipo de terapias muestra una emergente evidencia clínica sobre el abordaje del cáncer, el dolor crónico, los problemas cardiacos, trastornos depresivos, trastornos de ansiedad y trastornos de personalidad.

En la presente revisión, la mayor evidencia clínica procede de las Terapias basadas en Mindfulness (MBT); sin embargo, los resultados en población clínica son heterogéneos y limitados. Algunos estudios han mostrado resultados contradictorios (véase Morone, et al., 2008; Morone, et al., 2009) o una eficacia parcial (Mularski, et al., 2009; Robert-McComb, et al., 2004; Texeira, 2010). No obstante, las MBT han mostrado resultados prometedores en población mayor no clínica. En esta línea, se ha observado que la MBSR mejora el afecto positivo (Gallegos, et al., 2013), la percepción de soledad, disminuye al expresión de genes pro-inflamatorios (Creswell, et al., 2012) o la presión arterial (Palta, et al., 2012). Resultados similares se han encontrado en población joven (Carlson, Speca, Faris y Patel, 2007; Hoffman, Sawyer y Witt, 2010).

La Terapia de Aceptación y Compromiso (ACT) y la Activación Conductual (AC) son las aproximaciones terapéuticas que siguen en el número de evidencia empírica a las MBT. ACT muestra resultados significativos en la población mayor con diagnóstico clínico, en línea con la evidencia acumulada en otras poblaciones (Ruiz, 2010). Desde ACT, la evidencia clínica se centra en el dolor crónico, trastornos depresivos y de ansiedad. Sin embargo, carece de diseños controlados y/o seguimientos a largo plazo. Recientemente, se ha realizado un estudio sobre el impacto de un protocolo breve de ACT en población mayor con trastorno distímico y depresivo con un seguimiento de 5 y 12 meses (RuizSánchez, Cangas y Barbero, 2014). Si bien se trata de un diseño de caso único con tres réplicas, los resultados indican que una intervención breve e individual puede incrementar las acciones a valor en todos los participantes, manteniéndose tales acciones 12 meses tras la intervención. Este tipo de estudios, de los cuáles parte están emergiendo en España, abren una línea prometedora en la investigación sobre el impacto de ACT en el mayor.

Por otro lado, las terapias basadas en AC, se muestran eficaces en la población mayor con trastornos depresivos y trastorno por duelo. Esta terapia presenta datos sobre el efecto a largo plazo de la intervención mediante ensayos controlados. Actualmente, más estudios controlados desde la AC están en marcha (p.ej., Clignet, van Meijel, van Straten y Cuijpers, 2012).

La Terapia Dialéctico-Conductual (DBT) y la Terapia Analítico-Funcional (FAP) muestran menor evidencia, o ninguna en el caso de FAP, sobre sus efectos en la población mayor. Esto puede deberse principalmente a su interés en los trastornos de personalidad y la población adolescente. Si bien estas terapias, al igual que ACT, no están formuladas para una población particular, se hace nece- 
saria una mayor evidencia en este tipo de población.

En síntesis, la aplicación de este tipo de terapias está aumentando y muestra resultados prometedores en personas mayores con diagnóstico clínico. Sin embargo, la evidencia sigue siendo limitada y requiere un mayor número de ensayos clínicos controlados con seguimientos de larga duración.

\section{REFERENCIAS}

Acierno, R., Rheingold, A., Amstadter, A., Kurent, J., Amella, E., Resnick, H., ...Lejuez, C. (2012). Behavioral activation and therapeutic exposure for bereavement in older adults.American Journal of Hospice and Palliative Medicine, 29, 13-25.

Alonso, M., López,A., Losada, A., y González, J. L. (2013). Acceptance and commitment therapy and selective optimization with compensation for older people with chronic pain.Behavioral Psychology, 1, 59-79.

Areán, P. A., y Cook, B. L. (2002). Psychotherapy and combined psychotherapy/ pharmacotherapy for late life depression. Biological Psychiatry, 52, 293-303.

Bishop, S. R., Lau, M., Shapiro, S., Carlson, L., Anderson, N. D., Carmody, J., ...Devins, G. (2004).Mindfulness: A proposed operational definition. Clinical Psychology: Science and Practice, 11, 230-241.

Carlson, L.E., Speca, M., Patel, K.D., yFaris, P. (2007). One year follow-up of psychological, endocrine, immune and blood pressure outcomes of Mindfulness-Based Stress Reduction (MBSR) in breast and prostate cancer outpatients. Brain, Behavior and Immunity, 2, 1038-1049.

Clignet, F., van Meijel, B., van Straten, A., Lampe, I., yCuijpers, P. (2012).The Systematic Activation Method (SAM) in Depressed Elderly: A Case Report. Perspectives in Psychiatric Care, 48, 25-33.

Crane-Okada, R., Kiger, H., Sugerman, F., Uman, G. C., Shapiro, S. L., WymanMcGinty, W., y Anderson, N. L. (2012). Mindful movement program for older breast cancer survivors: a pilot study. Cancer Nursing, 35, E1-13.

Ekers, D., Webster, L.A., Van Straten, A., Cuijpers, P., Richards, D., yGilbody, S. (2014).Behavioural Activation for Depression: An Update of Meta-Analysis of Effectiveness and Sub Group Analysis.Plos One, 9, e100100.

Foulk, M. A., Ingersoll-Dayton, B., Kavanagh, J., Robinson, E., y Kales, H. C. (2013). Mindfulness-Based Cognitive Therapy With Older Adults: An Exploratory Study. Journal of Gerontological Social Work, 57, 498-520.

Gallagher, D. E., y Thompson, L. W. (1982). Treatment of major depressive disorder in older adult outpatients with brief psychotherapies.Psychotherapy: Theory, Research and Practice, 19, 482-490.

Gallagher, D. E., y Thompson, L. W. (1983). Effectiveness of psychotherapy for both endogenous and nonendogenous depression in older adult outpatients. Journal of Gerontology, 38, 707-712, 
Hayes, S. C. (2004). Acceptance and commitment therapy and the new behaviour therapies.En S. C. Hayes, V. M. Follette y M. M. Linehan (Eds.), Mindfulness and acceptance: Expanding thecognitive behavioral tradition (pp. 1-29).Nueva York: The Guilford Press.

Hayes, S. C., Strosahl, K. D., y Wilson, K. G. (1999). Acceptance and Commitment Therapy.AnExperiential Approach to Behavior Change.New York: Guilford Press.

Hofmann, S. G., Sawyer, A. T., Witt, A. A., y Oh, D. (2010). The effect of mindfulness-based therapy on anxiety and depression: A meta-analytic review. Journal of Consulting and ClinicalPsychology, 78, 169-183.

Jacobson, N. S., Dobson, K. S., Truax, P. A., Addis, M. E., Koerner, K., Gollan, J. K.,... Prince, S. E. (1996).A component analysis of cognitive-behavioral treatment for depression.Journal of Consulting and Clinical Psychology, 64, 295-304.

Jacobson, N. S., Martell, C. R., y Dimidjian, S. (2001).Behavioral activation treatment for depression: Returning to contextual roots. Clinical Psychology: Science and Practice, 8, 255-270

Kabat-Zinn, J. (1990). Full catastrophe living: Using the wisdom of your body and mind to face stress, pain and illness. New York: Delacorte.

Karlin, B. E., Walser, R. D., Yesavage, J., Zhang, A., Trockel, M., y Taylor, C. B. (2013). Effectiveness of Acceptance and Commitment Therapy for depression: Comparison among older and younger veterans. Aging \& Mental Health, 17, 555-563.

Kohlenberg, R. J., y Tsai, M. (1991). Functional analytic psychotherapy. New York: Plenum Press.

Kohlenberg, R. J., y Tsai, M. (1994). Functional Analytic Psychotherapy: A behavioral approach to treatment and integration. Journal of Psychotherapy Integration, 4, 175-201.

Linehan, M., M. (1993).Cognitive Behavioral Treatment of Borderline Personality Disorder. New York: Guilford Press.

Lynch, T. R., Cheavens, J. S., Cukrowicz, K. C., Thorp, S. R., Bronner, L., y Beyer, J. (2007). Treatment of older adults with co-morbid personality disorder and depression: a dialectical behavioral therapy approach. International Journal of Geriatric and Psychiatry, 22, 131-143.

Lynch, T. R., Morse, J. Q., Mendelson, T., yRobins, C. J.(2003).Dialectical behavior therapyfordepressed older adults: Arandomized pilot study. American Journal of Geriatric Psychiatry, 11, 1-13.

Luciano, C., y Valdivia, M. S. (2006). La Terapia de Aceptación y Compromiso (ACT). Fundamentos, características y evidencia. Papeles del Psicólogo, 27, 79-91.

Mackin, S. R., y Areán, P. A. (2005). Evidence-based psychotherapeutic Intervention for Geriatric Depression.PsychiatricClinic of North America, 28, 805-820.

Márquez-González, M., Fernández-Fernández, V., Romero-Moreno, R., y Losa- 
da, A. (2013). La terapia de aceptación y compromiso como procedimiento para optimizar las intervenciones potenciadoras del envejecimiento activo o exitoso. InformaciónPsicológica, 105, 42-59.

McCracken, L. M., y Jones, R. (2012). Treatment of chronic pain for adults in the seventh and eighth decades of life: A preliminary study of acceptance and commitment therapy (ACT). Pain Medicine, 13, 860-867.

Morone, N. E., Greco, C. M., y Weiner, D. K. (2008). Mindfulness meditation for the treatment of chronic low back pain in older adults : a randomized controlled pilot study. Pain, 134, 310-319.

Morone, N. E., Rollman, B. L., Moore, C. G., Qin, L., y Weiner, D. K. (2009). A mind-body program for older adults with chronic low back pain: Results of a pilot study. Pain Medicine, 10, 1395-1407.

Mularski, R., Munjas, B., Lorenz, K., Sun, S., Robertson, S., Schmelzer, W., ...Shekelle, P. G. (2009). Randomized controlled trial of mindfulness-based therapy for dyspnea in chronic obstructive lung disease.Journal of Alternative and ComplementaryMedicine, 15, 1083-1090.

Panos, P., Jackson, J., Hasan, O., y Panos, A. (2014). Meta-Analysis and Systematic Review Assessing the Efficacy of Dialectical Behavior Therapy (DBT). Research on Social Work Practice, 24, 213-223

Pérez-Álvarez, M. (2012).Third-Generation therapies: Achievements and challenges. International Journal of Clinical and Health Psychology, 12, 291-310.

Petkus, A. J., y Wetherell, J. L. (2013). Acceptance and commitment therapy with older adults: Rationale and considerations. Cognitive and BehavioralPractice, 20, 47-56.

Robert McComb, J .J.,Tacon, A., Randolph, P., y Caldera, Y. (2004). A pilot study to examine the effects of a mindfulness-based stress reduction and relaxation program on levels of stress hormones, physical functioning, and submaximal exercise responses.Journal of Alternative and Complementary Medicine, 10, 819-827.

Rosenthal, M. Z., Cheavens, J. S., Lejuez, C. W., y Lynch, T. R. (2005). Thought suppression mediates the relationship between negative affect and borderline personality disorder symptoms. Behaviour Research and Therapy, 43, 1173 - 1185.

Ruiz, F. J. (2010). A review of acceptance and commitment therapy (ACT). empirical evidence: Correlational, experimental psychopathology, component and outcome studies. International Journal of Psychology and Psychological Therapy, 10, 125-162.

Ruiz-Sánchez, L. J., Cangas, A. J., y Barbero, A. (2014). Intervención breve de Terapia de Aceptación y Compromiso (ACT) en ancianos institucionalizados con sintomatología depresiva. International Journal of Psychology and Psychological Therapy, 14, 445-458.

Segal, Z. V., Williams, J. M., y Teasdale, J. D. (2002). Mindfulness-based cognitive therapy for depression: A new approach to preventing relapse. New York: The Guilford Press. 
Teixeira, E. (2010). The effect of mindfulness meditation on painful diabetic peripheral neuropathy in adults older than 50 years.Holistic Nursing Practice, 24, 277-283.

Thompson, L. W., y Gallagher, D. (1984). Efficacy of psychotherapy in the treatment of late-life depression.Advances in Behavior Research Therapy, 6, $127-139$.

Wetherell, J. L., Afari, N., Ayers, C. R., Stoddard, J. A., Ruberg, J., Sorrell, J. T., ... Patterson, T. L. (2009). Acceptance and commitment therapy for generalized anxiety disorders in olders adults: A preliminary reports. BehaviorTherapy, 42, 127-134.

Wilson, K. G., y Luciano, C. (2002). Terapia de Aceptación y Compromiso: un tratamiento conductual orientado a los valores. Madrid: Pirámide. 